\title{
Distributed measurement of chromatic dispersion by four-wave mixing and Brillouin optical-time-domain analysis
}

\author{
Miguel González Herráez, Luc Thévenaz, and Philippe Robert \\ Metrology and Photonics Laboratory, École Polytechnique Federale de Lausanne, CH-1015, Lausanne, Switzerland
}

Received December 3, 2002

\begin{abstract}
A new nondestructive method for measuring the spatial distribution of chromatic dispersion along an optical fiber is presented. It is based on using Brillouin optical time-domain analysis to probe the power distribution of the four-wave mixing generated by two continuous-wave lasers. The results obtained prove that this new method is capable of providing better performance than comparable techniques. Furthermore, sensing the variations of Brillouin gain maximum produces additional information about the fiber, such as presence of strain and concentration of $\mathrm{GeO}_{2}$. (C) 2003 Optical Society of America

OCIS codes: $060.2270,060.4370,190.4380,190.5890$.
\end{abstract}

Soliton transmission in optical fibers requires tight control of local chromatic dispersion $D(z)$ along the optical fiber. ${ }^{1,2} \quad$ Several methods for mapping chromatic dispersion (CD) variations along the fiber have already been successfully developed. ${ }^{3-7}$ In this Letter we describe a new mapping method that overcomes several difficulties reported for previous configurations. It is based on sensing the power distribution of four-wave mixing (FWM) along the fiber by use of Brillouin optical time-domain analysis (BOTDA). Our method is based on the same effect as that described in Ref. 4 but avoids the problems encountered in this configuration that are due to modulational instability. Our method has the advantage of being suitable for mapping arbitrary values of CD coefficients, whereas those described in Refs. 3 and 5-7 are restricted to mapping the zero-dispersion wavelength of the fiber $\left(\lambda_{0}\right)$.

We consider two continuous-wave lasers $\left(\lambda_{I}\right.$ and $\left.\lambda_{K}\right)$ that deliver a few milliwatts $(5-10 \mathrm{~mW})$ of power at the fiber input $(z=0)$. We assume that the power of both lasers is below the Brillouin threshold. The wavelengths of the two lasers are separated by a quantity $\Delta \lambda$. By FWM, a Stokes wave $\left(\lambda_{S} \simeq \lambda_{K}+\Delta \lambda\right)$ and an anti-Stokes wave $\left(\lambda_{A} \simeq \lambda_{I}-\Delta \lambda\right)$ are generated. At a distance $z$ from the fiber input, the power of the anti-Stokes and the Stokes waves depends on the accumulated phase mismatch among the four waves involved in the process $\left(\Delta \beta=2 \beta_{I}-\beta_{K}-\beta_{A}\right.$ for the anti-Stokes wave), which in turn depends on the CD distribution along the fiber. Neglecting fiber losses and nonlinear pump depletion (this effect is negligible in our conditions), we can express the distribution of power of the anti-Stokes wave as

$$
P_{\mathrm{FWM}}(z) \propto \frac{1}{\Delta \beta^{2}} \sin ^{2}\left(\frac{\Delta \beta z}{2}\right),
$$

where, for small $\Delta \lambda$ (typically $1-4 \mathrm{~nm}$ ), $\Delta \beta$ can be written as a function of the dispersion:

$$
\Delta \beta=2 \pi c(\Delta \lambda / \lambda)^{2} D\left(\lambda_{I}\right) .
$$

Thus, for reasonable values of $\mathrm{CD}$, the power of the FWM fluctuates periodically along the fiber with a spatial frequency that can be linearly related to the CD.

The goal is, therefore, to measure the power distribution of the Stokes or the anti-Stokes wave along the fiber. With this power distribution and by a simple time-frequency analysis we should be able to extract the CD map along the fiber. A method for doing so has already been reported. ${ }^{4}$ Two strong $(\sim 1-\mathrm{W})$ pulses were launched at the input end of the fiber, and the oscillatory behavior of the FWM generated was recorded by use of the Rayleigh backscattered light at the Stokes or the anti-Stokes wavelength. The main problem with this technique is modulational instability if the CD is small and positive as a result of the large powers used. In our case, entirely different probing is used that is based on BOTDA. By contrast with the method described in Ref. 4, we benefit from the fact that Brillouin scattering is highly efficient in optical fibers, so our method can rely on continuous-wave, low-power $(\sim 10-\mathrm{mW})$ FWM pumps, which should be well below the threshold for modulational instability. Our theoretical analysis reveals that, as long as the Brillouin gain (BG) is not too large, the shape of the FWM evolution should be adequately retrieved, as can be straightforwardly shown by use of the firstorder approximation of the Brillouin amplification for counterpropagating pump pulse and signal waves:

$$
\Delta P_{A}(z)=\left(g / A_{\text {eff }}\right) P_{A}(z) P_{P}(z) \Delta z,
$$

where $g$ is the Brillouin linear gain, $A_{\text {eff }}$ is the mode effective area, $P_{A}$ and $P_{P}$ are the powers of the anti-Stokes and the Brillouin pump, respectively, and $\Delta z$ is the pump pulse length. Inasmuch as the Brillouin pump power is subject only to standard attenuation and thus varies slowly, the amplified part of the signal $\Delta P_{A}(z)$ is essentially proportional to the local power $P_{A}(z)$ of the anti-Stokes signal. As a result of the counterpropagating interaction between the waves, spatial distribution $\Delta P_{A}(z)$ can be retrieved directly from the time distribution of the anti-Stokes signal.

The experimental setup that we use to measure the FWM distribution along the fiber is depicted in Fig. 1. 


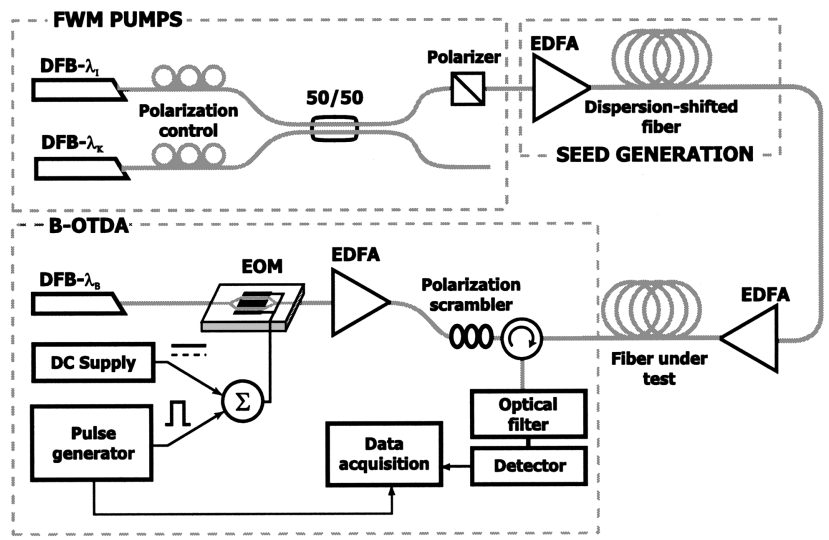

Fig. 1. Experimental setup: EOM, electro-optical modulator; EDFAs, erbium-doped fiber amplifiers. The BOTDA operates near $1555 \mathrm{~nm}$, and the pulse length is $1 \mu \mathrm{s}$.

Two temperature-tuned distributed-feedback lasers act as FWM pumps. The polarizations of the two lasers are carefully aligned, so the maximum FWM is obtained. Self- and cross-phase modulation effects among the FWM pumps are largely negligible because of the low powers used $(\sim 10 \mathrm{~mW})$. As a result of the interaction of the two FWM pumps, a Stokes (anti-Stokes) wave will be generated at frequency $f_{S}\left(f_{A}\right)$, following the model described above. The frequency of the BOTDA pump can be tuned to either $f_{S}+\nu_{B}$ or $f_{A}+\nu_{B}$, where $\nu_{B}$ is the Brillouin shift (see Fig. 2). Here the frequency of the BOTDA pump is tuned to $f_{A}+\nu_{B}$. Hence, following the arrangement described in Fig. 2, the system analyzes the CD at wavelength $\lambda_{I}$. We rough tune the frequencies by tuning the temperatures of the lasers, whereas we accomplish the fine tuning by modifying the current of the laser at $\lambda_{K}\left(I_{K}\right)$. To achieve a bigger signal in the fiber under test, generated a small $(<10-\mu \mathrm{W})$ coherent seed in a dispersion-shifted fiber just after the FWM pumps. Although it is small, this seed enhances the power oscillations within the fiber and greatly facilitates tuning of the instrument. The polarization of the BOTDA is scrambled to prevent polarizationdependent variation of the BG. We analyzed the resultant traces by computing the spectrogram in the range of frequencies that corresponds to the expected range of values for the dispersion. The width of the analysis window determines the final resolution and uncertainty of the map. Bigger windows ensure less uncertainty but also less resolution.

Figure 3 shows the trace obtained for a standard single-mode fiber. Our tests with standard fibers (with $\lambda_{0}$ in the second window) show that the relative variations in the $C D$ are less than the uncertainty of the instrument (approximately 5\% if only one period is taken in the analysis). This uncertainty is roughly similar to that reported in Ref. 4. We note that the resolution is given basically by the period of the spatial oscillation recorded. An improvement in the resolution demands an increase in $\Delta \lambda$ and thus a reduction in the contrast of the oscillations. Figure 4 shows a trace acquired for a nonzero dispersion-shifted fiber. In Fig. 5 we show the dispersion map of the previous fiber, computed by the previous method. The width of the window of analysis is large $(\simeq 2 \mathrm{~km}$, or $\sim 5$ signal periods) to ensure that a low degree of uncertainty is achieved. The mean error between the maps obtained from the two fiber ends is less than $0.8 \%$.

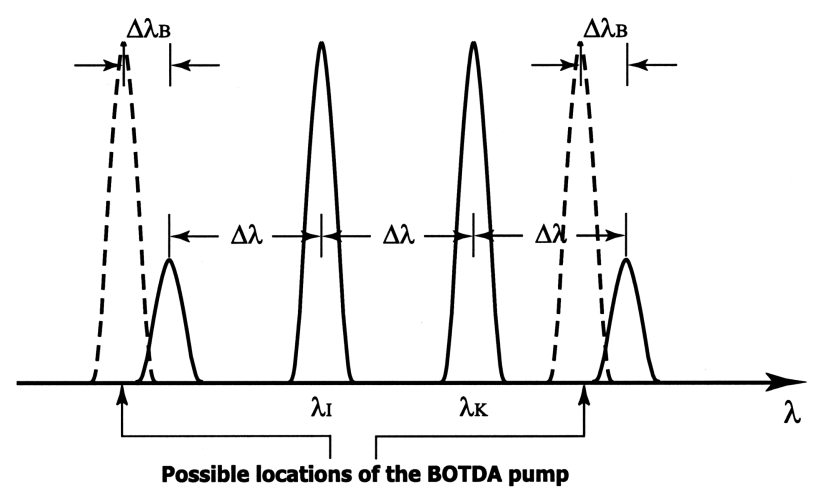

Fig. 2. Possible arrangement of the three wavelengths. $\Delta \lambda_{B}$ is the Brillouin shift.

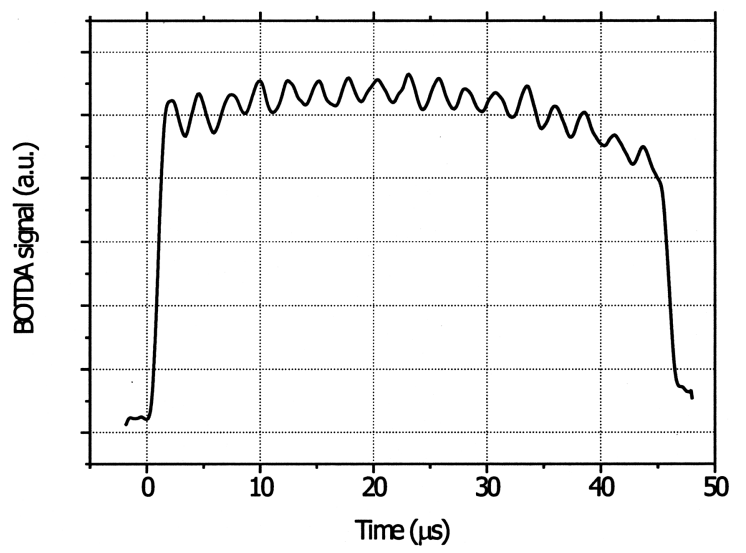

Fig. 3. BOTDA trace obtained for a $4.6-\mathrm{km}$ standard fiber with a CD of $16.2 \mathrm{ps} \mathrm{nm}^{-1} \mathrm{~km}^{-1}$ at the wavelength of the instrument. $\Delta \lambda=1.37 \mathrm{~nm}$. The period of the oscillation in this case is $\simeq 260 \mathrm{~m}$.

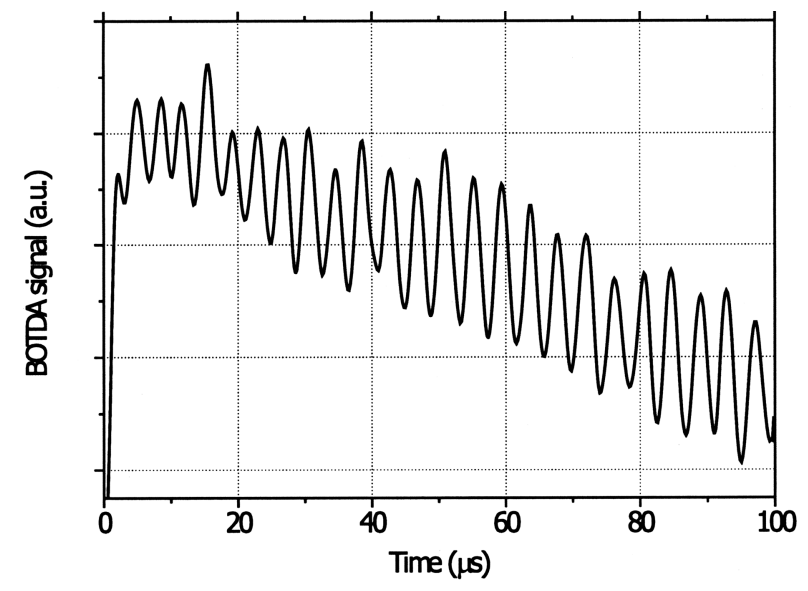

Fig. 4. Detail of the BOTDA trace obtained for a 10.34-km nonzero dispersion-shifted fiber with an end-to-end CD of $5.3 \mathrm{ps} \mathrm{nm}^{-1} \mathrm{~km}^{-1} . \Delta \lambda=1.96 \mathrm{~nm}$. The period of the oscillation in this case is $\simeq 390 \mathrm{~m}$. 


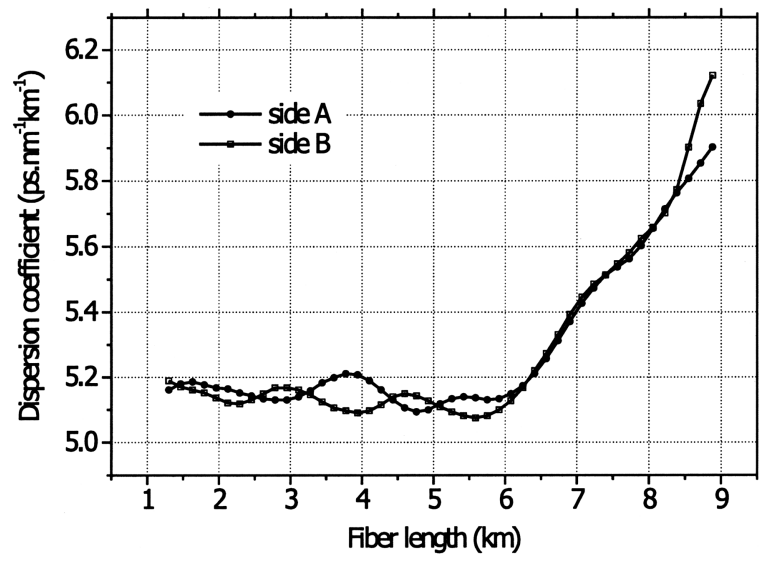

Fig. 5. CD map of the fiber whose trace appears in Fig. 4, computed from traces obtained from both fiber ends. The acquisitions were performed with $\Delta \lambda=2.12 \mathrm{~nm}$.

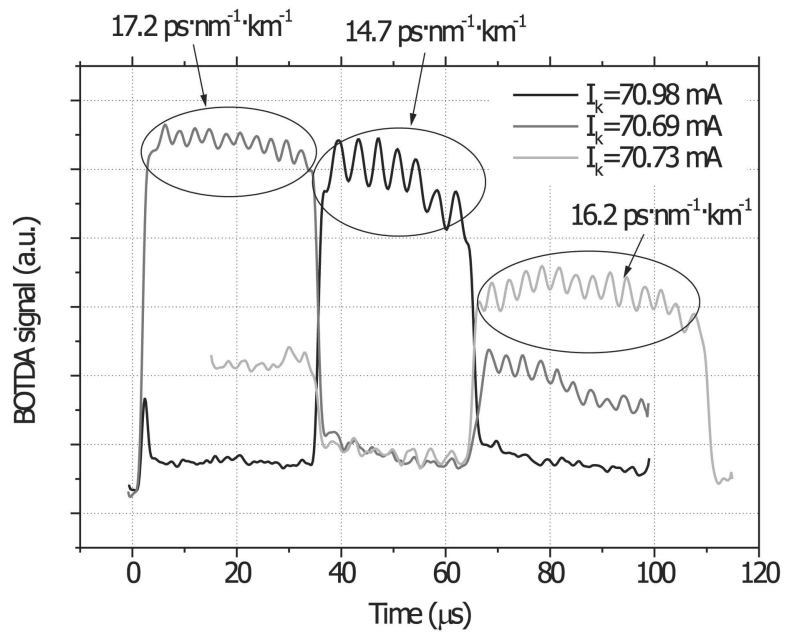

Fig. 6. Superposition of the traces obtained for a concatenation of three standard fibers for three values of $I_{K}$.

We note that our system can be used simultaneously to investigate whether there are variations in $\mathrm{GeO}_{2}$ concentration and to check for the presence of stress in the fiber. This information is related to the local shape and position of the BG curve. ${ }^{8}$ By slightly tuning the current $I_{K}$ and assuming that the frequency of the laser changes at a constant rate (typically $600 \mathrm{MHz} / \mathrm{mA}$ ), we can determine the relative variations of the BG maximum along the fiber and relate them to various local properties of the fiber. To illustrate this capability we spliced together three standard fibers with different dispersion coefficients and Brillouin shifts. Figure 6 shows the traces obtained for different values of $I_{K}$. Note that each fiber has a different Brillouin shift and a different CD but that both quantities can easily be determined from our measurements. It should be stressed that the measured $\mathrm{CD}$ and the nominal one (measured with an end-to-end technique) agree precisely. Note also that the contrast of the oscillations is higher as the dispersion is lower, in agreement with relation (1) and Eq. (2).

The main drawback of the present configuration is that the relative evolution of the three frequencies injected into the fiber (two FWM pumps and a Brillouin pump) has to be kept within the BG bandwidth of the fiber (typically $20-25 \mathrm{MHz}$ ). This fact implies that, with the present configuration, the system has to be retuned in periods not longer than a few minutes.

To sum up, we have demonstrated a new method of mapping chromatic dispersion in optical fibers. This new method overcomes many of the difficulties of other methods while it ensures good resolution and a low degree of uncertainty in the final determination of local CD values. Additionally, our system provides a way simultaneously to investigate variations of $\mathrm{GeO}_{2}$ and test for strain in the fiber. Further research will be needed to improve the relative stability of the three lasers to ensure that we are always working within the BG bandwidth. A straightforward improvement of the method might be obtained by use of slightly higher FWM pump powers (to increase $\Delta \lambda$ without losing contrast in the oscillations). This would require phase dithering of the FWM pumps (to overcome Brillouin scattering) in such a way that the generated FWM would still have a narrow linewidth. ${ }^{9}$

The authors acknowledge financial support from the Swiss Federal Office for Education and Science in the framework of the European COST265 action. M. González Herráez acknowledges support from the Comunidad Autónoma de Madrid. This research was conducted while M. González Herráez was on leave from the Instituto de Física Aplicada, Madrid, Spain; his e-mail address is ltqg340@cetef.csic.es.

\section{References}

1. M. Chertkov, I. Gabitov, P. M. Lushnikov, J. Moeser, and Z. Toroczkai, J. Opt. Soc. Am. B 19, 2538 (2002).

2. L. F. Mollenauer, P. V. Mamyshev, J. Gripp, M. J. Neubelt, N. Mamysheva, L. Grüner-Nielsen, and T. Veng, Opt. Lett. 25, 704 (2000).

3. S. Nishi and M. Saruwatari, Electron. Lett. 21, 579 (1996).

4. L. F. Mollenauer, P. V. Mamyshev, and M. J. Neubelt, Opt. Lett. 21, 1724 (1996).

5. I. Brener, P. P. Mitra, D. D. Lee, D. J. Thomson, and D. L. Philen, Opt. Lett. 23, 1520 (1998).

6. M. Eiselt, R. M. Jopson, and R. H. Stolen, J. Lightwave Technol. 15, 135 (1997).

7. M. González-Herráez, P. Corredera, M. L. Hernanz, and J. A. Méndez, Opt. Lett. 27, 1546 (2002).

8. M. Niklès, L. Thévenaz, and Ph. Robert, J. Lightwave Technol. 15, 1842 (1997).

9. M. C. Ho, M. Marhic, K. Y. K. Wong, and L. G. Kazovsky, J. Lightwave Technol. 20, 469 (2002). 